\title{
The Variation of Radon Exhalation Rates from Concrete Surfaces of Different Ages
}

\author{
K. N. YU* \\ E. C. M. YOUNG* \\ T. F. CHAN* \\ T. LO† \\ R. V. BALENDRAN†
}

\author{
(Received 31 March 1995; accepted 8 December 1995)
}

\begin{abstract}
The radon exhalation rates from four concrete blocks cast with ordinary Portland cement $(O P C)$ and four with substitution of pulverized fuel ash $(P F A)$ were monitored for 34 months starting from November 1992. At the end of the 28 th month, two OPC blocks and two PFA blocks were immersed in water, one from each group for 5 days the other one from each group for 10 days. These were then taken out of water and the radon exhalation rates monitored as usual. It was observed that the radon exhalation rate in general decreased with the age of the concrete blocks and the rate increased after the blocks were immersed in water. An explanation for the first result is that the gradual dehydration of concrete as it ages will reduce the water content in the pores of the concrete, thus reducing the probability of retaining radon within the pores and the probability of radon emanation from these pores. The second result gives strong support to this assertion. Copyright (C) 1996 Elsevier Science Ltd.
\end{abstract}

\section{INTRODUCTION}

IN a recent latitudinal study on the variation of radon exhalation rates from concrete surfaces of buildings in Hong Kong with the age of the concrete, the radon exhalation rate was found to decrease with age [1]. Since suggestions regarding the variation of radon exhalation rates with water content have been pul forward for concrete [2] and for soil [3], it is natural to assert that the phenomenon observed in the latitudinal study is also due to the change of water content of the concrete over time [1].

There were three objectives in the present investigation. The first was to perform a long-term longitudinal study of the radon exhalation rate from concrete surfaces. Four concrete blocks were cast with ordinary Portland cement (referred to as OPC blocks) in the laboratory, which had fixed radium contents and provided opportunities for a longitudinal study. The second objective was to identify any similar behaviour in concrete blocks cast with OPC substituted with pulverized fuel ash (PFA). Four concrete blocks were cast with OPC substituted with 15\% PFA (referred to as PFA blocks) in the laboratory for the longitudinal study. The third objective was to assess the assertion that the decrease in radon exhalation rates from concrete surfaces with time is due to the gradual dehy-

*Department of Physics and Materials Science, City University of Hong Kong, Tat Chee Avenue, Kowloon Tong, Hong Kong.

$\dagger$ Department of Building and Construction, City University of Hong Kong, Tat Chec Avenue, Kowloon Tong, Hong Kong. dration of concrete, which reduces the water content in the pores of the concrete, thus reducing the probability of radon retention within the pores and the probability of radon emanation from these pores. At some point of the monitoring programme of the radon exhalation rates, selected OPC blocks and PFA blocks were immersed in water, and then taken out of water for the identification of any enhancement of radon exhalation.

\section{METHODOLOGY}

The method for the measurement of the radon exhalation rates from concrete surfaces was described by [1] Standardized charcoal canisters [4] were used to collect the radon exhaled for two to three days. These were sealed against the concrete surfaces with silicone sealer to stop air leakage. After collection, the charcoal canisters were removed from the surface, sealed, and stored to allow the radon decay to reach equilibrium. The radon activities inside the canisters were then determined by counting the gamma ray photons emitted by the radon decay products inside at energies of 295, 352 and $609 \mathrm{keV}$ using an $\mathrm{NaI}$ gamma spectrometer for 10 minutes.

The radon exhalation rate $\epsilon$ (in units of Bq. $\mathrm{m}^{-2} \cdot \mathrm{s}^{-1}$ ) can be calculated using [1]

$$
\epsilon=\frac{\lambda\left(\mathrm{NET}^{2} \mathrm{e}^{\lambda t}\right.}{S E\left(1-\mathrm{e}^{-\lambda T}\right) 3600},
$$

where $\lambda\left(=0.00756 \mathrm{~h}^{-1}\right)$ is the physical decay constant of radon, NET is the net photon count rate (cpm) at the three energies mentioned above, $S$ is the area of the 


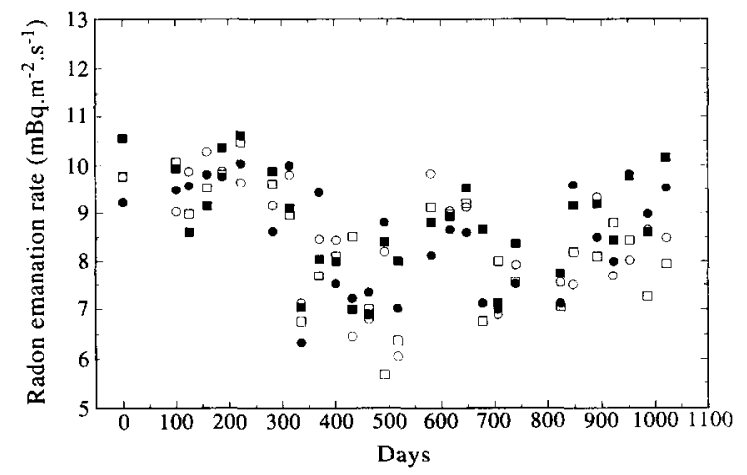

Fig. 1. The temporal variation of radon exhalation rate (mBq. $\mathrm{m}^{-2} \cdot \mathrm{s}^{-1}$ ) from OPC concrete blocks. Solid circle: OPC sample 1; solid square: OPC sample 2; outline circle: OPC sample 3; outline square: OPC sample 4. Sample 1 was immersed in water during the period from the 834th to the 839th day and sample 2 from the 828 th to the 838 th day.

concrete surface covered by the charcoal canister, $E$ is the detection efficiency of the system, $T$ is the radon collection time, $t$ is the time elapsed from the end of collection to the start of measurements, and 3600 is the conversion factor from hours to seconds.

The radon exhalation rate from surfaces of all the concrete blocks was monitored for 34 months (November 1992 to August 1995) in a laboratory kept at a temperature of $23^{\circ} \mathrm{C}$ and a relative humidity of about $60 \%$. For each measurement, three adjacent surfaces of each concrete block were measured to eliminate the efffect of the possible uneven distribution of granite inside the concrete blocks. These three surfaces were used for the whole course of the investigation.

\section{RESULTS AND DISCUSSION}

The experimental results of the measurements of radon exhalation rates from the OPC and PFA concrete blocks are shown in Figs 1 and 2. It should be noted that all the concrete blocks were cast on 1 November 1992, but the zeroth day was set to the date of the first measurement (i.e. 11 November 1992) for convenience. Therefore, the actual age of the concrete blocks should be obtained by adding 10 days to the number shown on the $x$-axis of

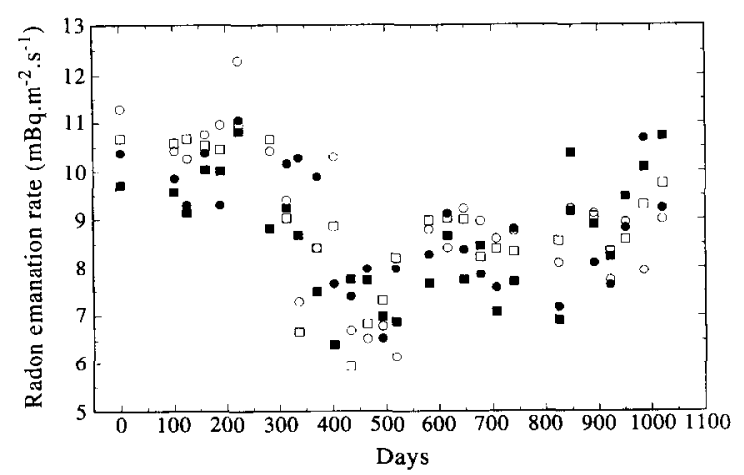

Fig. 2. The temporal variation of radon exhalation rate (mBq. $\mathrm{m}^{-2} \cdot \mathrm{s}^{-1}$ ) from PFA concrete blocks. Solid circle: PFA sample 1; solid square: PFA sample 2; outline circle: PFA sample 3; outline square: PFA sample 4. Sample 1 was immersed in water during the period from the 834th to the 839th day and sample 2 from the 828 th to the 838 th day.

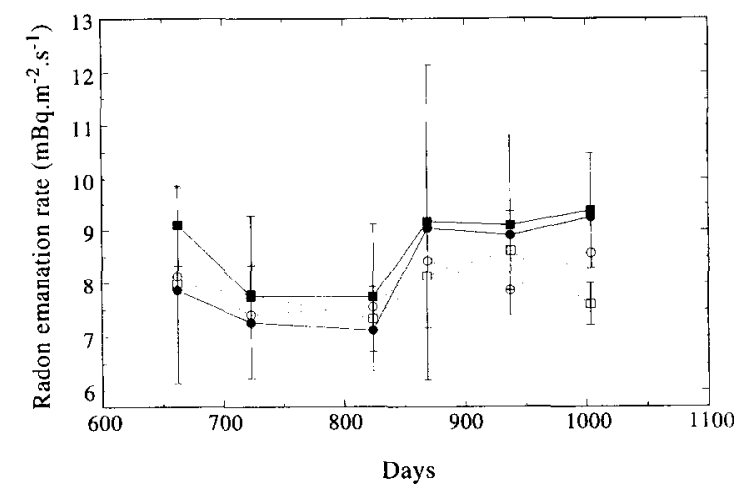

Fig. 3. The temporal variation of radon exhalation rate (mBq. $\mathrm{m}^{-2} \cdot \mathrm{s}^{-1}$ ) from OPC concrete blocks. Solid circle: OPC sample 1; solid square: OPC sample 2; outline circle: OPC sample 3; outline square: OPC sample 4. Sample 1 was immersed in water during the period from the 834 th to the 839 th day and sample 2 from the 828 th to the 838 th day. For reference, the third set of data shown in this figure was collected on the 824th day.

Figs 1 and 2. The OPC sample 1 and the PFA sample 1 were immersed in water during the period from the 834th to the 839 th day, and the OPC sample 2 and PF $\wedge$ sample 2 from the 828 th to the 838 th day. Therefore it should be noted that there is a data divide which separates those before the 828th day from those after the 839 th day.

From Fig. 1, an anti-correlation between the radon exhalation rate and the age of OPC concrete blocks can be seen to the left of the divide, although there are evidently some fluctuations which have also been observed elsewhere $[5,6]$. The solid symbols refer to the data for the concrete blocks which were immersed in water during the divide period, while the outline symbols refer to the data for the undisturbed concrete blocks. It seems that the outline symbols can extend beyond the divide but the solid symbols do not and rise above their projected values, which means that the OPC blocks have enhanced radon exhalation after being immersed in water. Similar behaviour for the PFA blocks can be inferred from Fig. 2 , although the fluctuations are larger here.

To illustrate more clearly the enhancement of radon

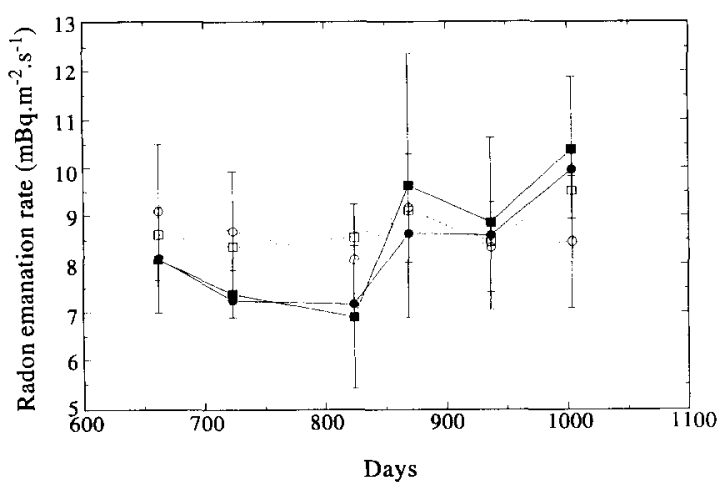

Fig. 4. The temporal variation of radon exhalation rate (mBq.m $\mathrm{m}^{-2} \cdot \mathrm{s}^{-1}$ ) from PFA concrete blocks. Solid circle: PFA sample 1; solid square: PFA sample 2; outline circle: PFA sample 3; outline square: PFA sample 4. Sample 1 was immersed in water during the period from the 834 th to the 839 th day and sample 2 from the 828 th to the 838 th day. For reference, the third set of data shown in this figure was collected on the 824th day. 
exhalation, we focus on the data in a relatively short period before and after the immersion of concrete blocks in water. For this purpose, data with close dates have been pooled together to smooth out short-term fluctuations. The results for OPC blocks are shown in Fig. 3 and those for PFA blocks are shown in Fig. 4. In Figs 3 and 4, there are six sets of data. Except for the third set of data, all the data represent the average of two consecutive measurements separated by about 1 month, and the day is taken to be the middle of the period. The third set of data represent a single measurement because there are no measurements on a close date. The immersion of the selected concrete blocks took place between the third and the fourth sets of data. It is therefore clearly seen that the radon exhalations are enhanced.
The general decrease of radon exhalation rates from concrete surfaces with time can be explained by the progressive dehydration of concrete, which reduces the water content in the pores of the concrete, thus reducing the probability of retaining radon within the pores and the probability of radon emanation from these pores [2, 3]. Our results showing an enhancement of the radon exhalation from concrete blocks immersed in water give strong support to this assertion.

Acknowledgement-This research is supported in part by research grant No. 700426 from the City University of Hong Kong.

\section{REFERENCES}

1. K. N. Yu, T. F. Chan and E. C. M. Young, The variation of radon exhalation rates from building surfaces of different ages. Health Physics 68, 716-718 (1995)

2. E. Stranden, A. K. Kolstad and R. Lind, Radon emanation: moisture and temperature dependence Health Physics 47, 480-484 (1984).

3. R. L. Fleischer, Moisture and ${ }^{222}$ Rn emanation. Health Physics 52, 797-799 (1987).

4. B. L. Cohen and E. S. Cohen, Theory and practice of radon monitoring with charcoal adsorption. Health Physics 45, 501-508 (1983).

5. K. Ulbak, N. Jonassen and K. Bækmark, Radon exhalation from samples of concrete with different porositics and fly-ash additives. Radiation Protection Dosimetry 7, 45-48 (1984).

6. L. M. M. Roelofs and L. C. Scholten, The effect of aging, humidity and fly-ash additive on the radon exhalation from concrete. Health Physics 67, 266-271 (1994). 